\title{
AN EXTENSION OF THE CHIRP SCALING ALGORITHM TO 3-D NEAR-FIELD WIDE-BAND RADAR IMAGING
}

\author{
E. Gimeno-Nieves , C. Pascual , J. M. Lopez-Sanchez \\ Dept. de Física, Ingeniería de Sistemas y Teoría de la Señal (DFISTS) \\ University of Alicante, P.O.Box 99, E-03080, Alicante, Spain \\ Tel: +34 965909673, Fax: +34 965903464, E-mail: encarna@ disc.ua.es
}

\begin{abstract}
The objective of the present work is the development of an efficient algorithm to focus two-dimensional SAR images under the following extreme conditions: large coherent integration angles and high bandwidth-to-centrefrequency ratios $\left(B / f_{c}\right)$. These conditions constitute a common situation in wide-band high-resolution SAR configurations.

The algorithm presented in this paper is an extension of the well-known chirp scaling algorithm (CSA). First, the original 2-D CSA [1] is generalized by extending the formulation from data obtained with a linear aperture to data measured by a planar synthetic aperture. Moreover, a new compact generalized formula of the impulse response function in the three-dimensional frequency domain is presented. These extensions enable the development of a new 3 -D algorithm in the aforementioned conditions. Finally, the algorithm has been implemented and satisfactory tested.
\end{abstract}

\section{INTRODUCTION}

Synthetic aperture radar (SAR) [2] is used for the generation of reflectivity images of scenes illuminated by microwave pulsed signals. The generation of such images requires the coherent combination of the echoes recorded by the SAR sensor at uniformly spaced positions in order to focus all the targets properly. In this way, the final azimuth resolution in the images is similar to that obtained with a large antenna.

The problem of focusing SAR data is usually studied by considering the signal received from a single point scatterer. So, the system is fully described by a model of its impulse response function (IRF). The IRF is very useful to formulate the focusing procedure of real scenes, which in turn are treated as a superposition of point scatterers.

Focusing algorithms have to deal with the well-known effects of range curvature and the variation of this curvature over the range. These effects are accurately compensated in conventional air and space-borne configurations. However, the design of the focusing procedure is more demanding under any of the following extreme conditions:

1. A large coherent integration angle.

2. A low center-frequency-to-bandwidth ratio, or quality factor $(Q)$.

The first condition is common when working with highresolution radar configurations and, in particular, with ground-based systems and anechoic chambers. The second condition appears in penetrating SAR systems for detection of concealed targets. Hereafter, we refer to these conditions as near-field wide-band conditions.

A similar problem is common in high-resolution synthetic aperture sonar (SAS) configurations. SAS systems operate in the kilohertz region and present a low- $Q$ nature [3]. Therefore, the image processing requirements of SAR (under the conditions shown before) and SAS are equivalent. The main purposes of SAS are sea floor and sub-bottom imaging and, also, to detect proud, tethered and buried mines.

A precise method to achieve excellent accuracy in nearfield wide-band conditions is the so-called range migration algorithm (RMA), or $\omega-\mathrm{k}[4,5]$. The drawback of this technique is the introduction of an interpolation stage that implies a high computational cost and slows down the image generation. As an alternative, the chirp-scaling algorithm (CSA) $[2,1]$ avoids this interpolation, since its implementation requires only Fourier Transforms and complex products. Therefore, it is more efficient than the RMA. The original CSA produces high quality images when applied to standard space and air-borne SAR systems. However, the generation of accurate images by the original CSA in near-field wide-band conditions is limited by the approximations assumed in its formulation, even for targets located at the reference range which the algorithm is tuned for.

The key point is that standard CSA formulations provide only up to the second or third order of the Taylor series expansion of the IRF phase in the rangefrequency/azimuth-frequency domain [6], and higher terms are neither considered nor presented. The incorporation of additional terms (up to $n$ th-order) makes it possible to maintain the efficiency of the method and to improve considerably its accuracy in near-field wide-band conditions. So, the main contributions of the present work are:

- The extension of the original CSA to the 3-D case of a planar aperture.

- A new general expression of the IRF phase for chirped signals in the 3-D frequency domain, that enables the improvement of radar and sonar imaging techniques under near-field wide-band conditions.

\section{FORMULATION}

Starting with the conventional formulation of the CSA, an analytical expression of the IRF in the frequency domain can be derived in a three-stage procedure $[1,2]$. Consider the measurement set-up shown in Fig. 1. We suppose a chirp modulation radar with a chirp rate $\Gamma$ and a wavelength $\lambda$ at the center frequency. The antenna is positioned 


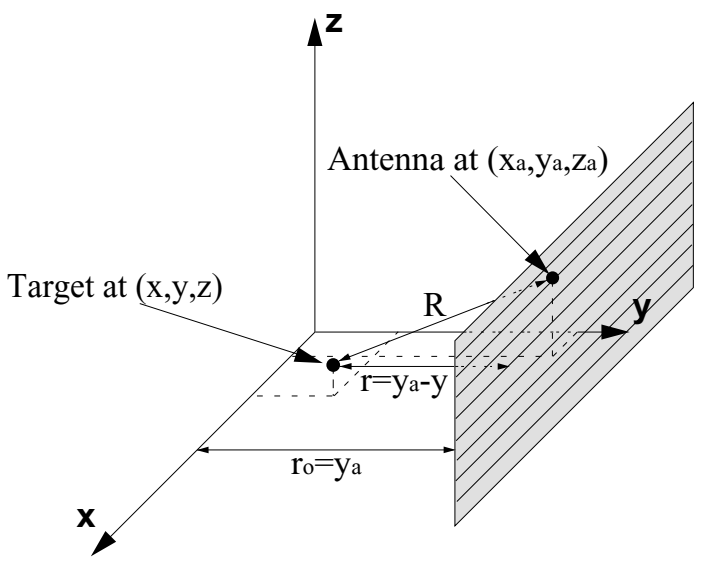

Figure 1: Measurement and three-dimensional imaging geometry.

at $\left(x_{a}, y_{a}, z_{a}\right)=\left(x_{a}, y_{a c}, z_{a}\right)$ coordinates and it synthesizes a planar aperture of size $L_{x} \times L_{z}$ at a distance $r_{0}$ from the scene center and centered at $\left(x_{a c}, y_{a c}, z_{a z}\right)$. By assuming that there is a point scatterer located at $(x, y, z)$ with reflectivity 1 , the analytic expression of the baseband signal impulse response $s\left(\tau, x_{a}, z_{a} ; r\right)$ can be derived:

$$
\begin{aligned}
s\left(\tau, x_{a}, z_{a} ; r\right) & =s_{a}\left(x_{a}, z_{a}\right) \cdot s_{r}\left(\tau-\frac{2}{c} R\left(x_{a}, z_{a} ; r\right)\right) \\
& \cdot \exp \left(-j \pi \Gamma\left(\tau-\frac{2}{c} R\left(x_{a}, z_{a} ; r\right)\right)^{2}\right) \\
& \cdot \exp \left(-j \frac{4 \pi}{\lambda} R\left(x_{a}, z_{a} ; r\right)\right),
\end{aligned}
$$

where $\tau$ is time in the slant direction and $r$ is the slant range from the point scatterer. The function $s_{a}(\cdot)$ is the azimuth aperture-weighting pattern, $s_{r}(\cdot)$ is the transmitted signal envelope, and $R\left(x_{a}, z_{a} ; r\right)$ is the instantaneous distance between the sensor and the point scatterer. The constant $c$ is the velocity of the light. Note that we have approximated the wavenumber in the slant range direction by the constant $\frac{4 \pi}{\lambda}$.

The formulation begins with the range Fourier transform of this signal impulse response by applying the method of stationary phase (MSP), followed by an azimuth Fourier transform of the resulting expression (again by MSP). Finally, the point scatterer phase response in the frequency domain,

$$
\begin{aligned}
& \Phi_{I R F}\left(k_{r}, k_{x}, k_{z} ; r\right)=-k_{x} x-k_{z} z+\cdots \\
& +r_{o} k_{r}+\frac{c^{2}}{16 \pi \Gamma} k_{r}^{2}+\cdots \\
& +\frac{-4 \pi r}{\lambda}\left[A_{x z}^{2}\left(k_{x}, k_{z}\right)+\frac{\lambda}{2 \pi} k_{r}+\left(\frac{\lambda}{4 \pi}\right)^{2} k_{r}^{2}\right]^{1 / 2},
\end{aligned}
$$

where

$$
A_{x z}\left(k_{x}, k_{z}\right)=\left[1-\left(\frac{\lambda k_{x}}{4 \pi}\right)^{2}-\left(\frac{\lambda k_{z}}{4 \pi}\right)^{2}\right]^{\frac{1}{2}}
$$

can be replaced by its Taylor's series expansion. Here we present for the first time an $n$ th-order expression of this expansion:

$$
\begin{aligned}
& \Phi_{I R F}\left(k_{r}, k_{x}, k_{z} ; r\right)=\sum_{i=0}^{\infty} \phi_{k}\left(k_{x}, k_{z} ; r\right) k_{r}^{i} \simeq \\
& \simeq \sum_{i=0}^{n} \phi_{k}\left(k_{x}, k_{z} ; r\right) k_{r}^{i}= \\
& =-k_{x} x-k_{z} z-\frac{4 \pi}{\lambda} r A_{x z}\left(k_{x}, k_{z}\right)+\cdots \\
& +\left(r_{o}-\frac{r}{A_{x z}\left(k_{x}, k_{z}\right)}\right) k_{r}+\frac{c^{2}}{16 \pi \Gamma_{s}\left(k_{x}, k_{z} ; r\right)} k_{r}^{2}+\cdots \\
& +\sum_{i=3}^{n} \frac{r}{2^{i}}\left(\frac{\lambda}{4 \pi}\right)^{i-1} G_{i}\left(k_{x}, k_{z}\right) k_{r}^{i},
\end{aligned}
$$

where

$$
\Gamma_{s}\left(k_{x}, k_{z} ; r\right)=\frac{\Gamma}{1+\Gamma r \frac{2 \lambda}{c^{2}} \frac{\left(\frac{\lambda k_{x}}{4 \pi}\right)^{2}+\left(\frac{\lambda k_{z}}{4 \pi}\right)^{2}}{\left[1-\left(\frac{\lambda k_{x}}{4 \pi}\right)^{2}-\left(\frac{\lambda k_{z}}{4 \pi}\right)^{2}\right]^{\frac{3}{2}}}},
$$

and

$$
G_{i}\left(k_{x}, k_{z}\right)=\sum_{j=\operatorname{ceil}\left(\frac{i}{2}\right)}^{i} \frac{(-1)^{j} 2^{j} \cdot \Pi_{p=1}^{j-1}(2 p-1)}{A_{x z}^{2 j-1}\left(k_{x}, k_{z}\right) \cdot(2 j-i) !(i-j) !} .
$$

The operator $\operatorname{ceil}(i / 2)$ rounds $i / 2$ to the nearest integer towards infinity. The variables $k_{r}, k_{x}$ and $k_{z}$ are the wavenumber of range and azimuth (cross-range) directions, respectively.

Regularly, we can discard cubic and higher order terms of (4), but in near-field wide-band conditions we have to consider some higher order terms than squared to compensate the extra range curvature produced by this configuration. Indeed, this expression enables to control the approximation error by a phase function multiplier in the rangefrequency/azimuth-frequency domain (see Fig. 2). Results will prove the utility of this new formula in the generation of images with excellent radiometric and geometric accuracy.

The near-field wide-band algorithm consists in transforming the chirped SAR data into the frequency domain with a Fast Fourier Transform (FFT) and introducing a new multiplier phase function that compensates the rest of required terms in the Taylor's expansion at a reference range. The required image accuracy and the importance of the near-field wide-band situation determine the highest term in this phase function. Since these high order terms are not coupled with the rest of phase functions, the remaining steps are equivalent to the original CSA.

For stepped-frequency radar, the signal data are in the range-frequency domain and they are not chirped. In that case, the data are first transformed to the 3-D frequency domain, and then a chirp operation and the near-field wideband correction can be performed at the same complex product. 


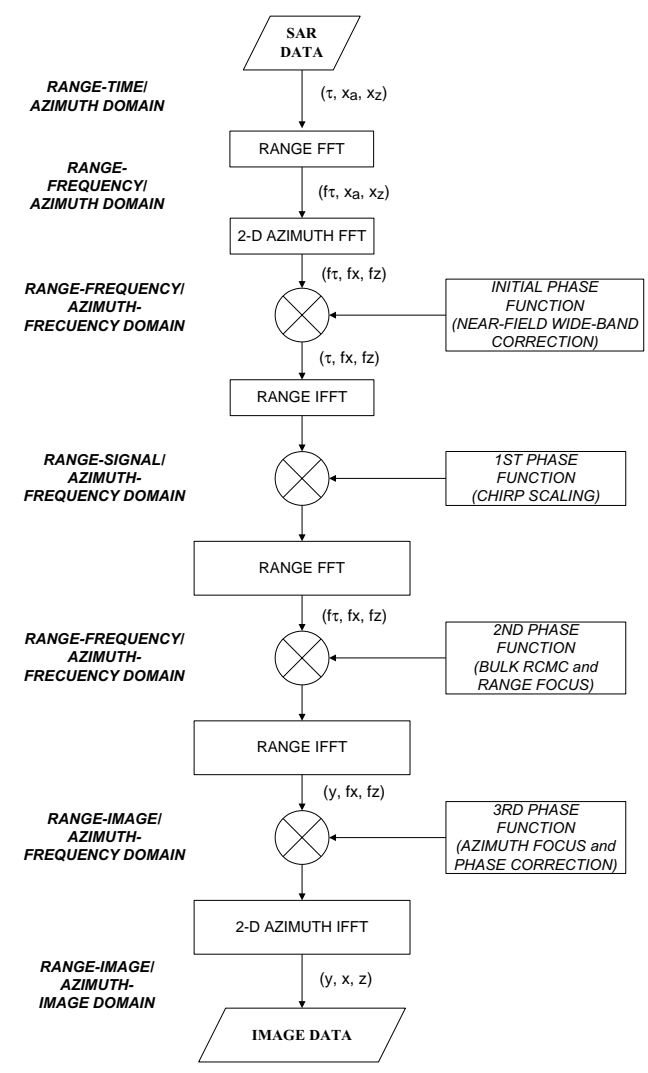

Figure 2: Flow diagram of the near-field wide-band 3-D CSA for chirped data.

\section{RESULTS}

Some simulations were carried out in order to verify the improvement produced by canceling higher orders of the phase in the frequency domain than in the original CSA. For the sake of clarity, we reduce our analysis to the 2-D case (linear aperture) and simulate a single point scatterer at the center of the scene. The length of the aperture is $2 \mathrm{~m}$, and it is located at $2 \mathrm{~m}$ from the target. Frequency ranges from 2 to $6 \mathrm{GHz}$. Note that this configuration produces an extreme integration angle and a quite low quality factor $(Q=1)$. For comparison purposes, some two-dimensional images have been obtained with the original CSA and the near-field wide-band CSA including extra terms from the third order to the seventh order.

Figure 3 shows two orthogonal cuts of the image crossing at the scatterer position. In the cut at constant range, shown in Fig. 3(a), we can observe that the width of the main lobe gets smaller when we increase the maximum order in the series expansion. On the other hand, the cut at constant azimuth in Fig. 3(b) exhibits that the first secondary lobes are not at the same level, and, as the order increases, this difference begins to disappear. We can also deduce from these results that an optimum order can be found for any configuration.

The performance of this new algorithm has been also tested experimentally by employing data from the outdoor linear SAR system called LISA, developed at the European Microwave Signature Laboratory (EMSL) at JRC Ispra, Italy. This system is based on a stepped frequency radar

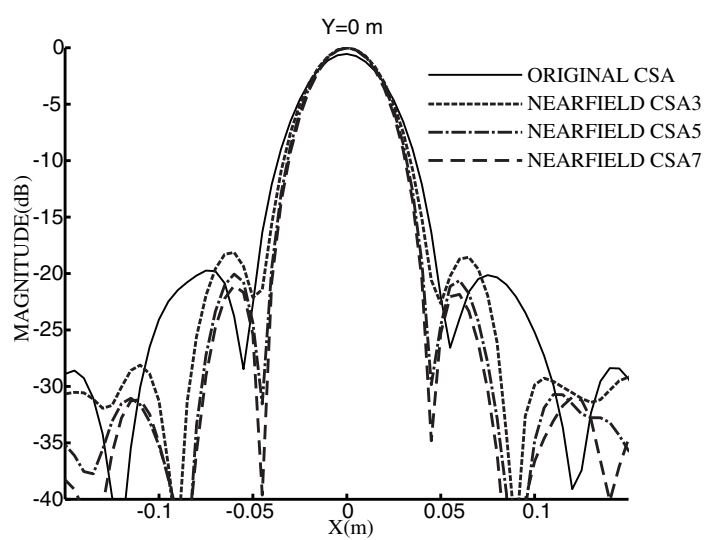

(a)

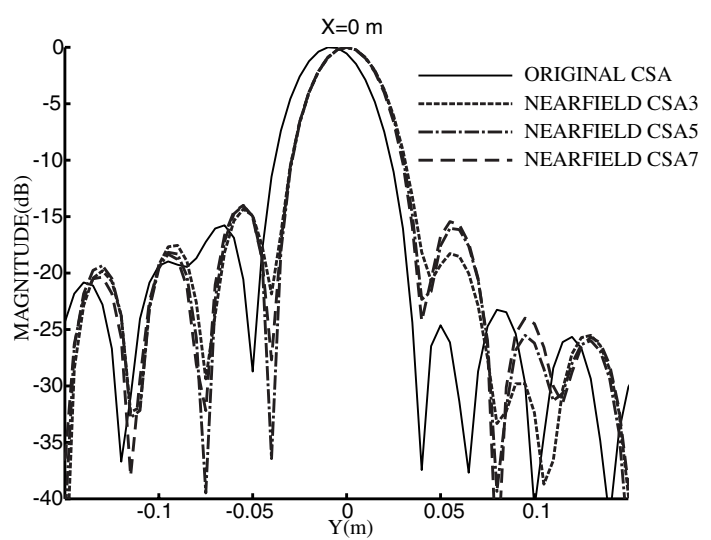

(b)

Figure 3: 2-D point scatterer response of SAR system: (a) at constant range $y=0,(b)$ at constant azimuth $x=0$.

equipped with a 2-D positioning system. The target consists of a 3-D arrangement of eight metallic spheres of diameter $7.62 \mathrm{~cm}$. The dimensions of the synthetic aperture are $1 \mathrm{~m} \times 1 \mathrm{~m}$. Frequency ranges from 15.5 to $17.5 \mathrm{GHz}$. The distance from the center of the aperture to the scene center is $2.3 \mathrm{~m}$. The plane of the aperture is tilted 14 degrees from the vertical direction (see Fig. 4(a)). The theoretical resolutions are in this case $2 \mathrm{~cm}$ in the horizontal and vertical cross-range directions, and $7.5 \mathrm{~cm}$ in the ground-range direction.

Figure 4(b) shows several slices out of the reconstructed $3-\mathrm{D}$ image. The displayed dynamic range is $20 \mathrm{~dB}$. As expected, the reflectivity at the positions of the spheres is about $-23.4 \mathrm{dBsm}$, corresponding to RCS given by the physical optics approximation. The measured spatial resolutions are in agreement with the expected ones. Note that the reflectivity peaks of the spheres closer to the antennas are narrower because the effective synthetic aperture is larger in the near range. On the other hand, the spheres have a diameter of about four wavelengths and therefore they are not ideal point scatterers. As a result, a minor degradation or defocusing must be expected.

It is important to note that this data set was already used [5] to validate the 3-D RMA. Consequently, we can perform here a comparison between these two techniques. 

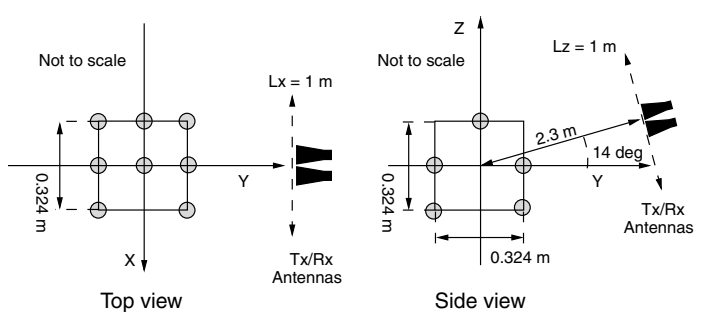

(a)
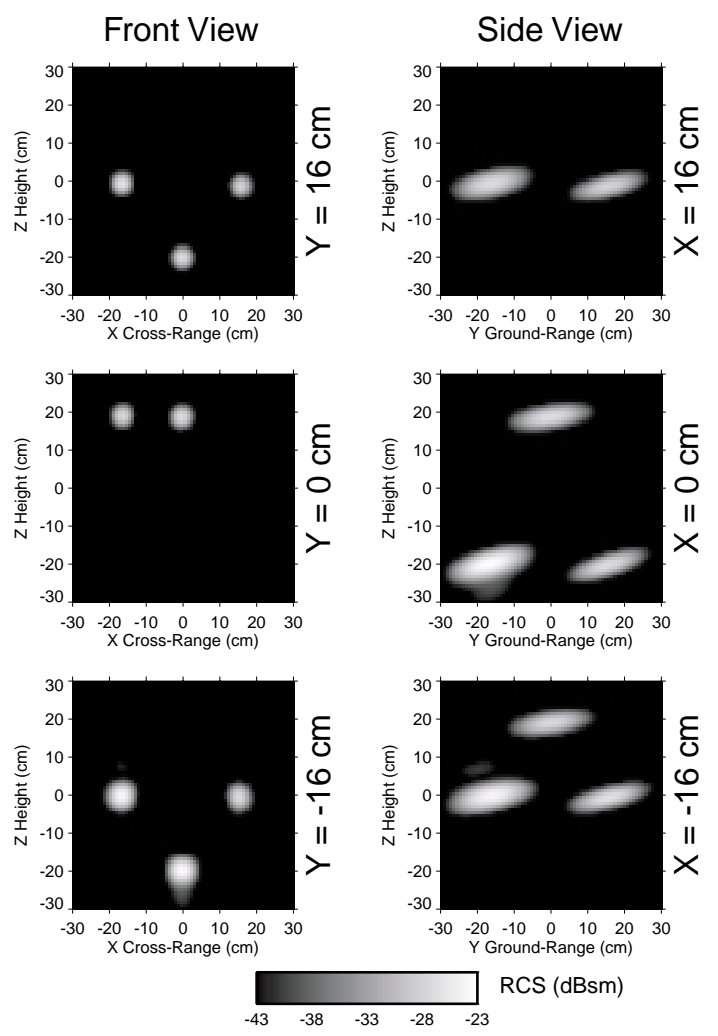

(b)

Figure 4: Experiment. (a) Measurement set-up used in the experimental validation with a 3-D array of eight spheres. (b) Slices of the reconstructed 3-D image.

In principle, the near-field CSA is not as accurate as the RMA due to the approximations assumed in the formulation. However, even in the extreme conditions presented in these tests, its performance is accurate enough for most applications. With respect to the computation time, we have run both algorithms in the same platform for comparison purposes. The 3-D RMA took 82 seconds to generate the reflectivity image, whereas the new near-field 3-D CSA only took 65 seconds. This time reduction is provided by the substitution of the Stolt interpolation (the bottleneck of the RMA) by Fourier transforms and complex products.

\section{CONCLUSIONS}

For the first time, an $n$ th-order general expression of the phase impulse response of a synthetic aperture system in the two-dimensional frequency domain has been presented. Thanks to this contribution, additional terms have been formulated and included in the CSA to obtain more accurate images in near-field wide-band configurations. The number of extra terms to be incorporated depends on the particular configuration and geometry of the measurement, and it can be freely selected in the algorithm because an $n$ th-order expression is now available.

The algorithm has been implemented and tested with simulations. An evident improvement has been demonstrated by observing the shape and magnitude of the reconstructed reflectivity of a point scatterer. Numerical simulations have also shown that the dynamic range is better than 70-80 dB, even in extreme conditions.

Furthermore, the new algorithm can be combined with other extensions of the CSA proposed before by other authors $[6,7]$. Finally, it is important to note that this extension of the algorithm can be applied to any synthetic aperture system, such as sonar and radar.

\section{ACKNOWLEDGMENT}

This work was supported by the Generalitat Valenciana under Project No. GV00-036-14 and the Spanish Commission of Science and Technology (CICYT) under Project No. TIC2001-2364-C03-02.

\section{REFERENCES}

[1] R. K. Raney, H. Runge, R. Bamler, I. G. Cumming and F. H. Wong: "Precision SAR Processing Using Chirp Scaling”, IEEE Trans. Geosci. Remote Sensing, Vol. 32, July 1994, pp. 786-799.

[2] W. G. Carrara, R. S. Goodman and R. M. Majewski: "Spotlight Synthetic Aperture Radar. Signal Processing Algorithms", Artech House, 1995.

[3] P. T. Gough and D. W. Hawkins: "A short history of synthetic aperture sonar", IEEE Int. Geosc. Remote Sensing Symposium IGARSS'98, Seattle, 6-10 July 1998.

[4] C. Cafforio, C. Prati and E. Rocca: "SAR Data Focusing Using Seismic Migration Techniques”, IEEE Trans. Aerospace Electronic Syst., Vol. 27, No. 2, Mar. 1991, pp. 194-207.

[5] J. M. Lopez-Sanchez and J. Fortuny: "3-D Radar Imaging Using Range Migration Techniques”, IEEE Trans. Antennas Propagat., Vol. 48, May 2000, pp. 728-737.

[6] G. W. Davidson, I. Cumming and M. R. Ito: "A Chirp Scaling Approach for Processing Squint Mode SAR Data”, IEEE Trans. Aerospace Electronic Syst., Vol. 32,Jan. 1996, pp. 121-133.

[7] A. Moreira, J. Mittermayer and R. Scheiber: "Extended Chirp Scaling Algorithm for Air- and Spaceborne SAR Data Processing in Stripmap and ScanSAR Imaging Modes", IEEE Trans. Geosci. Remote Sensing, Vol. 34,Sept. 1996, pp. 1123-1136. 